\title{
Nestedness and turnover of riverine species and functional diversity using eDNA and traditional approaches
}

Mathew Seymour ( $\square$ mat.seymour@gmail.com )

Swedish University of Agricultural Sciences https://orcid.org/0000-0002-3654-4857

\section{François Edwards}

$\mathrm{CEH}$

Jack Cosby

NERC Centre for Ecology \& Hydrology

Pete Scarlet

$\mathrm{CEH}$

lliana Bista

Sanger

Francesca Brailsford

Bangor University

Helen Glanville

Bangor University

\section{Mark de Bruyn}

University of Sydney https://orcid.org/0000-0003-1528-9604

\section{Gary Carvalho}

University of Wales at Bangor

\section{Simon Creer}

Bangor University https://orcid.org/0000-0003-3124-3550

\section{Article}

Keywords: COI, macroinvertebrates, chironomids, EPT, functional diversity

Posted Date: October 6th, 2020

DOl: https://doi.org/10.21203/rs.3.rs-82612/v2

License: (c) (1) This work is licensed under a Creative Commons Attribution 4.0 International License.

Read Full License 


\section{Abstract}

Accurately assessing community diversity in time and space, and linking these patterns to ecological theory, is essential for effective environmental monitoring. Freshwater macroinvertebrates are an important group of taxa routinely used for riverine environmental assessments due to their wide biological, functional and phylogenetic diversity and their responses to environmental factors. Recently, eDNA metabarcoding based sampling and identification has been shown to increase the accuracy of biodiversity assessments, while reducing cost and time, compared to traditional methods. Here, we present results from a field comparison of eDNA versus traditional riverine biodiversity techniques to assess freshwater macroinvertebrates. In addition, we investigated the effects of landuse and seasonality on community and functional diversity, to infer the underlying regional ecological temporal and spatial dynamics. Comparison of biodiversity dynamics based on traditional and eDNA survey methods showed significant differences in taxonomic groups identified between methods, landuse type, and method $\mathrm{x}$ season interactions. Our findings are the first example of eDNA derived functional spatio-temporal and dynamics, indicating that the regional shifts in diversity and function are linked to regional seasonal fluctuations in fine particle matter versus localized landuse type. Beta diversity components (nestedness and turnover) differed significantly along the environmental gradient, but in different directions, for each methodology. Overall, our findings show that eDNA based ecological assessment is effective in assessing temporal and spatial diversity and functional dynamics of macroinvertebrates, while demonstrating that these data can be used to infer effective biodiversity assessment and management strategies.

\section{Introduction}

Our understanding of the processes that shape community assembly and the underlying maintenance of biodiversity is essential for the effective management of ecosystems and associated ecosystem services 1 . Community assembly is driven by multiple factors, including abiotic filtering, biotic interactions, historical context, dispersal limitation and stochastic processes ${ }^{2-4}$. Habitat or environmental filtering can drive the observed pattern of functional diversity within a community, such that the functional traits of a community reflect the selective forces induced by the local abiotic or biotic factors. While environmental filtering has been shown to play a crucial role on local or wide biogeographic scales, the regional (e.g. a single river catchment) observations needed for disentangling abiotic and biotic patterns are still largely underrepresented ${ }^{2}$. River catchments are a crucial component of regional biodiversity that harbor high levels of diversity due to their hierarchical structure, and the connectivity of environmentally diverse habitats within a single catchment ${ }^{5-7}$. Spatial and temporal changes in river biodiversity are also important indicators of regional environmental health, which is also heavily influenced by, and sensitive to, human activity ${ }^{8}$. However, current bioassessment practices do not routinely assess communities simultaneously for biotic interactions or changes in functionality across seasons, which can cause reduced representation of spatial or temporal dynamics needed to inform management strategies. In order to elucidate the relationship between biodiversity and ecosystem health we need to utilize 
increasingly more robust and cost-effective methodologies that allow routine and extensive assessment of community dynamics through time and space.

Changes in community composition can be monitored in many different ways, including through assessment of richness (i.e. alpha diversity), or functional diversity. Richness is defined as the number of unique taxonomic units per site/sample. Richness is often positively correlated with heterogeneous environments, which is often attributed to higher levels of functional diversity ${ }^{9-11}$. On the other hand, functional diversity quantifies the functionally disparate taxa within a community, and is becoming increasingly recognized as an important component of effective biomonitoring ${ }^{12}$. The most widely used functional assessment for macroinvertebrates involves organic matter decomposition, characterization whereby macroinvertebrates are classified as either shredders, collectors, or grazers/scrapers, though other groupings are also used to further separate functionality ${ }^{13}$. Additionally, inter-community structure is also important in assessing how assemblages change over time. Commonly referred to as beta diversity, inter-community structure can be further assessed to determine if communities are locally unique (nested), or if the community dissimilarity between sites is driven by replacement of species from other sites (turnover) ${ }^{14}$. Partitioning the variance into nested and turnover components provides greater insight into the processes that are driving inter-community homogenization or dissimilarity ${ }^{15}$.

Current biomonitoring practices in rivers utilize biological indices derived from freshwater macroinvertebrates. Freshwater macroinvertebrates represent a wide range of species and functional groups, which respond dynamically to temporal and spatial environmental filtering, Taxonomic identification of freshwater macroinvertebrates for biomonitoring is largely limited to mature life stages that and can be difficult among taxonomically similar species or genera. The high level of taxonomic specialization required to identify specimens and the long processing times per traditional sample rendering large scale ecosystem-wide traditional assessments expensive and time consuming ${ }^{16}$. The application of environmental DNA (eDNA) and metabarcoding, have been recently shown to offer increased sampling resolution for biodiversity assessment efforts ${ }^{17}$. Environmental DNA is extracted directly from an environment sample (for example, water, soil or air) without prior isolation of the organisms themselves ${ }^{18,19}$. Sources of eDNA include sloughed skin cells, urine, feces, saliva or other bodily secretions, and could consist of both free molecules (extracellular DNA) and free cells ${ }^{20-23}$. Furthermore, eDNA collected from water samples has highly sensitive detection capability, is non-invasive to the sampled biota and not limited to physical environmental conditions (e.g. surface area or substrate types), thereby providing a wider sampling application with lower environmental impact compared to traditional methods. Combined with high throughput sequencing (HTS) applications, such as metabarcoding, eDNA based sampling is rapidly being integrated into standard ecological monitoring practices. Previous eDNA biomonitoring studies in natural environments have assessed population and communities across spatial and temporal scales for rivers ${ }^{24-26}$, lakes ${ }^{27-29}$ and marine environments ${ }^{30-}$ 32 . What is currently lacking is the link between functional and community diversity dynamics using eDNA across appropriate spatial and temporal scales. However, we can hypothesize possible links 
between functional and community dynamics from existing ecological principles and recent eDNA research.

Here we assess seasonal patterns of biodiversity and functional diversity using an experimental design which utilizes headwater sampling sites to associate local environmental conditions located within the same environmentally heterogeneous geographic region (e.g. catchment). We investigate whether metabarcoding of headwater riverine eDNA is effective for the assessment of macroinvertebrate community diversity dynamics across season and landuse types. With regards to seasonal communities of riverine macroinvertebrates, richness or alpha diversity, is expected to peak during spring and summer, when many stream macroinvertebrates are emerging as adults and reproducing, compared to fall and winter months when total biomass of many species has declined ${ }^{27,33}$. Environmental filtering effects that are linked to habitat modification, such as agriculture or urbanized areas, are expected to negatively impact macroinvertebrate diversity, particularly Chironomidae and Ephemeroptera, Plecoptera and Trichoptera (EPT) taxa ${ }^{34}$, and lead to homogenized functional diversity ${ }^{35}$ through the combined processes of environmental filtering and biotic interactions ${ }^{2,3}$. Utilizing the nested and turnover components of inter-community similarity (i.e. beta-diversity), we can expect high turnover within sites as community assembly changes over time, and high nestedness across environmental sites, attributed to environmental filtering of the localized sites. Alternatively, low nestedness could indicate a low effect of environmental filtering and a greater effect of stochastic or biotic factors influencing the localized community assemblies.

\section{Methods}

\section{Study Area}

The Conwy Catchment is a $678 \mathrm{~km}^{2}$ river drainage in north Wales that encompasses a wide range of habitats including forest, moorland, agriculture, light urbanization, and acid grasslands (Fig. 1). The area experiences rapid climatic shifts, particularly during winter months due to its mountainous terrain and porous rock foundations, which facilitates flash flooding, making it susceptible to periodic disturbance ${ }^{36}$. The Conwy Catchment area exhibits four distinct seasons that correspond to the expected life cycles of EPT and Chironomidae larval emergences throughout the year.

\section{Sampling}

Fourteen sites across 5 landuse types (acid grassland, agriculture, forest, urban, moorland) were sampled once per season (spring, summer, fall, winter), during 2017 ( 5 landuse types $\times 14$ sites $\times 4$ seasons). In total 168 eDNA samples and 56 traditional kick-net samples were taken over the course of the study. Sampling for each season occurred over two consecutive days. For all sampling events, streams were sampled for both eDNA and macroinvertebrate community composition during the same day. Water samples for eDNA analysis $(1 \mathrm{~L})$ were collected in triplicates from each stream with plastic bottles that had been cleaned prior using a 10\% bleach solution. These were then filtered through $0.22 \mu \mathrm{m}$ SterivexTM 
filter units (EMD Millipore Corporation, Billerica, USA) using a Geopump TM Series II peristaltic pump (Geotech, Denver, USA). The filters were immediately preserved in ATL lysis buffer (Qiagen, Venlo, The Netherlands) and stored at room temperature during transit to the laboratory for further processing. Macroinvertebrate communities were sampled using a standardized 3-minute kick sampling protocol, with a $500 \mu \mathrm{m}$ mesh gauge kick/hand net. Both bank margins and riffle habitats were sampled during this timed sampling period. Macroinvertebrates were preserved in absolute ethanol (99.8\%; VWR International, Lutterworth, UK) on collection. Upon return to the laboratory the macroinvertebrates were cleared of other collected material and identified to the lowest practical taxonomic level. Environmental (abiotic) data including $\mathrm{pH}$, conductivity, depth, moss coverage, algal coverage, plant coverage boulder coverage, gravel coverage and sand coverage, were collected for each site following UK Environment Agency site assessment protocols ${ }^{37}$. Three field blanks consisting of deionized water were prepared in Nalgene sampling bottles and were kept with the other sampling gear throughout each sampling event. One blank was processed after every fourth sampling site ( 3 per sampling event; 12 blanks total) and treated the filters in the same manner as the other samples throughout the subsequent extraction and sequencing protocols.

\section{Extraction and Sequencing}

DNA was extracted from the filters using a modified QIAGEN DNA blood and tissue extraction protocol ${ }^{38}$. In short proteinase $\mathrm{K}$ was added directly to the filters and incubated overnight in a rotating hybridization chamber. Then, the lysate was extracted and the full volume was filtered through a spin column tube, after which point the standard extraction protocol was continued. Extracts were then cleaned for impurities using QIAGEN Power Clean kit and frozen at $-20^{\circ} \mathrm{C}$ for subsequent analyses. Sequencing libraries were created using a two-step protocol (see Bista et al. 2017), using matching dual end index tags (IDT) and the following COI gene region primers for the first round of PCR (PCR1): m1COlintF (5'GGWACWGGWTGAACWGTWTAYCCYCC-3') and jgHCO2198 (5'-TAIACYTCIGGRTGICCRAARAAYCA-3') ${ }^{39}$. Libraries were created at Bangor with the assistance of a Gilson pipette max liquid handler before being shipped to University of Birmingham's Genomic sequencing facility for quality control and sequencing. Round 1 amplification (PCR1) with the COI primers was performed in triplicates, which were then pooled and index labeled during Round 2 PCR step (PCR2). PCR1 utilized Thermo Scientific's Ampli-gold mastermix Due to the high number of inosine in the COI primer pair, and for PCR2 we utilized New England Biolab's Q5 mastermix. All PCR1 and PCR2 reactions were run in $25 \mu \mathrm{L}$ volumes. PCR1 amplicons were generated using a reaction mix of $12.5 \mu \mathrm{L}$ mastermix, $2 \mu \mathrm{L}$ DNA template, $1 \mu \mathrm{L}$ of each primer and $8 \mu \mathrm{L}$ nuclease free water and amplified using an initial $95^{\circ} \mathrm{C}$ for 5 min then 25 cycles of $95^{\circ} \mathrm{C}$ for $30 \mathrm{~s}, 54^{\circ} \mathrm{C}$ for $30 \mathrm{~s}$ and $72{ }^{\circ} \mathrm{C}$ for $60 \mathrm{~s}$ followed by a $72{ }^{\circ} \mathrm{C}$ final annealing for $10 \mathrm{~min}$. PCR2 amplicons were generated using a reaction mix of $12.5 \mu \mathrm{L}$ mastermix, $2 \mu \mathrm{L}$ DNA template, $1 \mu \mathrm{L}$ of each primer and 8 $\mu \mathrm{L}$ nuclease free water and amplified using an initial $98^{\circ} \mathrm{C}$ for $30 \mathrm{~s}$ then 15 cycles of $98^{\circ} \mathrm{C}$ for $10 \mathrm{~s}, 55^{\circ} \mathrm{C}$ for $30 \mathrm{~s}$ and $72{ }^{\circ} \mathrm{C}$ for $30 \mathrm{~s}$ followed by a $72^{\circ} \mathrm{C}$ final annealing for $10 \mathrm{~min}$. The, PCR2 amplicons were purified using High Prep PCR magnetic beads (Auto Q Biosciences) and quantified using a 200 pro plate reader (TECAN) with the QubitQubit dsDNA HS kit (Invitrogen). The final amplicons were pooled in 
equimolar quantities (at a final concentration of $12 \mathrm{pmol}$ ) using a Biomek FXp liquid handling robot (Beckman Coulter). Pool molarity was confirmed using a HS D1000 Tapestation ScreenTape (Agilent). Sequencing was performed on an Illumina HiSeq platform 250bp Paired-End, with an intended coverage of 100,000 reads per sample.

\section{Bioinformatics}

Bioinformatic processing up to taxonomic assignment was performed by University of Birmingham. In short, per base quality trimming was performed on demultiplexed reads using SolexaQA++ v.3.1.7.1 (Cox, Peterson, \& Biggs, 2010) and paired end reads were merged using Flash v.1.2.11 40 , with default parameters. Primer sequences were removed with TagCleaner v.0.16 ${ }^{41}$ allowing up to 3 mismatches per primer sequence. Only sequences with both forward and reverse primers were retained for further analyses. Operational Taxonomic Units (OTUs) were obtained via Usearch at $97 \%$ similarity threshold, and denoising with the -unoise3 algorithm. Taxonomy was assigned to representative OTU with BLAST against OTUs using the non-redundant nucleotide database of NCBI.

After stringent filtering and quality control, 12,592,362 reads were obtained with an average of 74,954 (SD $=31,050)$ reads per sample. Negative controls, while showing no bands on agarose gels post library preparation, generated 676 reads across all blanks $(\mathrm{N}=12)$. Of the negative control reads, 411 were unknown bacteria, 3 reads were associated with three genera of Rhodophyta (red algae), 2 reads were linked to unknown fungi, and 260 reads were linked to a single Dipteran OTU across four blanks.

\section{Metacommunity diversity measures}

All statistical analyses were performed using $\mathrm{R}$ version 3.6.1 ${ }^{42}$. Sequence reads were rarified for each set of replicates to the lowest replicate level. Mean number of reads for each OTU were calculated across the rarified data before being matched to their taxonomic identifier. Genera richness was calculated as the number of unique genera per site. We further divided richness into unique EPT genera for EPT richness, and Chironomidae richness as the number of unique Chironomidae genera. Functional richness was calculated as the number of unique functional groups per sample, following the designation of functional groups in Moog (2017). These groups reflect the species' trophic function, divided into 8 categories, including; grazer/scrapers, xylophagous, shredders, gatherers/collectors, active filter feeders, passive filter feeders, predators and other. We refined these groups to shredders, grazers, and collectors whereby collectors were the summation of gather/collectors and filter feeding groups to simplify the functional groups to those used across wider studies. Beta diversity was calculated as nestedness and turnover components following Baselga (2010).

\section{Statistics}

We used linear regression, as implemented using the Im function in $\mathrm{R}$, to assess the statistical relationships between (1) community, (2) EPT, (3) Chironomidae, and (4) functional richness (each as a separate response variable) and all two-way interactions of the explanatory variables, including sampling 
method (eDNA or traditional), season (spring, summer, fall and winter) and landuse gradient (see below for description). We further used linear regression to assess the statistical relationships between nestedness and turnover for the community, EPT and Chironomidae groupings (as response variables) against a set of explanatory variables, including the landuse gradient, sampling method, and season, including all possible two-way interactions for each model. The landscape gradient was calculated as the first principle component of a PCA derived from non-covarying environmental variables, where noncovariance was assessed via visualization of the pairwise correlation between all measured environmental variables (Fig S1) (Borcard, Gillet, \& Legendre, 2011). Backward model selection was performed to find the most parsimonious model using Akaike information criterion (AIC) to determine the best model fit ${ }^{43}$. Model assumptions, including normality and heteroscedasticity using model diagnostic plots were implemented in R. To assess the potential of spatial autocorrelation in the data, we calculated Moran's I for each subset of the data used to perform the above-mentioned regressions. Moran's I is a measure of spatial autocorrelation and was calculated via the ape package in $\mathrm{R}^{43}$.

\section{Results}

All environmental variables and their associated summary statistics are presented in Table 1 \& Figure 2 . Variation in genera alpha, Chironomidae and EPT richness is presented in Figure 3. The full breakdown of genera per landuse type can be found in supplementary table S1. The Moran's I test for spatial autocorrelation was non-significant for the eDNA sampling including alpha diversity $(p=0.89)$, as well as the Chironomidae $(p=0.78)$ and EPT $(p=0.11)$ subsets. The traditional sampling, however did show spatial autocorrelation for the alpha $(p<0.01)$ and EPT $(p<0.01)$ data, but not for the Chironomidae $(p=$ 0.78 ) subset.

\section{Methods comparison}

Genera richness derived from eDNA was significantly greater than traditional derived eDNA across season $(p<0.01)$ and landuse type $(p<0.001)$. There was a significant landuse $x$ method interaction effect $(p<0.01)$ and season $x$ method effect indicating non-covarying biodiversity dynamics between the two methods. Results for EPT also indicated non-covarying biodiversity dynamics with significant landuse $x$ method $(p<0.001)$, and method $x$ season $(p<0.001)$, whereas both methods showed significant differences across seasons and landuses. Chironomidae diversity also showed significant landuse $x$ method $(p<0.001)$ and method $x$ season $(p<0.001)$ interactions as well as significant effects of landuse and seasons on richness dynamics. Functional diversity showed significant landuse $x$ method $(p<0.001)$ and method $x$ season $(p<0.001)$ interactions, as well as seasonal $(p<0.001)($ Table 2 \& Fig. 3$)$.

Nestedness was non-significant across landuse for genera derived from eDNA $(p=0.241)$ or traditional methods $(p=0.072)$. There was a significant negative effect of landuse on genera turnover for eDNA $(p=0.003)$ and a significant positive effect of landuse on traditional $(p<0.001)$ taxa assignment. EPT nestedness was non-significant for eDNA $(p=0.893)$ and traditional methods $(p=0.576)$, whereas EPT turnover was significantly negative in relation to landuse for eDNA $(p=0.010)$ and significantly positive for 
traditional methods $(p<0.001)$. Chironomidae nestedness was non-significant for both eDNA $(p=0.287)$ and traditional methods $(p=0.640)$, with turnover non-significant for eDNA $(p=0.056)$ and positively significant from traditional methods $(p=0.016)$ (Table 3$)$. Positive and negative relationships with regards to landuse relate to the environmental gradient depicted in Fig S1 and described above.

\section{Change in community and functional diversity over time and space}

Changes in communities over time were directly related to the change in functionality over time (Fig. 4). For eDNA samples the increase in overall functionality was largely driven by the increase in number of genera from Diptera (all landuses), Coleoptera (Acid grasslands, Moorlands, Urban and Agriculture), Ephemeroptera (Forest), and Trichoptera (Forest and Moorlands). Transitioning into summer, acid grasslands and moorlands showed a loss of scraper and collector functionality stemming from losses in Plecoptera and Trichoptera genera. Losses of Plecoptera genera in forest resulted in a loss of scraper functionality, whereas gains in Plecoptera in the urban environments showed gains in collector functionality. Agriculture sites showed gains in scraper and gatherer function, due to some increases in Diptera and Trichoptera. Transitioning into fall indicated loss in functionality across landuse, driven by losses in Diptera (all landuses), Coleoptera (all landuses), Ephemeroptera (agriculture, moorland), and Trichoptera (urban, forest, agriculture). Winter was largely static, with the exception of gains in functionality for agriculture sites, which was driven by increased occurrence of Plecoptera and Trichoptera genera. Traditional samples indicated slight increases in functionality for agriculture and moorland landuses, primarily from increases in Plecoptera (agriculture, urban) and Coleoptera (moorland) genera. Summer increases in functionality for acid grasslands and moorlands were linked to increases in Trichoptera and Plecoptera in acid grasslands, and Plecoptera and Coleoptera in moorlands. Declines in fall gatherer function, in agriculture and forest landuses, was driven by losses in Trichoptera (agriculture sites) and Plecoptera (forest sites). Loss of functionality in winter for acid grassland, agriculture and moorlands was driven by losses in Trichoptera (acid grasslands), Plecoptera (acid grasslands, moorlands), Ephemeroptera (acid grasslands, agriculture, moorland), and Coleoptera (acid grasslands).

\section{Discussion}

Understanding the dynamics of biodiversity in time and space is essential for effective management of natural environments. Here we show how biodiversity patterns derived from eDNA and traditional methods show similar temporal trends in richness and functional diversity. However, beta diversity dynamics differed, particularly with regards to turnover across landuse types. Biodiversity estimates differed between sampling methods, with greater diversity and sensitivity to environmental conditions being observed when using eDNA based richness, compared to richness derived from traditional methods. Importantly we show here that eDNA based biodiversity shows meaningful spatial and temporal relationships, including increased ability to detect important indicator taxa, particularly Chironomidae and EPT taxa, which are difficult to directly sample for many of the locations or at different timepoints in the year. We also show the first example of eDNA derived functional temporal spatial dynamics, with direct links to the associated richness and beta-diversity dynamics. Furthermore, by 
combining findings from eDNA and traditional methods we can gain insights into the biodiversity dynamics that shape regional functional diversity and inter-community diversity.

These findings support our expectations that alpha diversity was greater during spring and summer months for both sampling methods. Genera diversity dynamics differed between eDNA and traditional methods among landuse types, but not among seasons where eDNA derived diversity was consistently greater than traditional diversity. In particular, we found greater eDNA derived diversity compared to traditional methods for all sites. Additionally, across landuse types, acid grasslands and moorland sites had greater eDNA diversity compared to agriculture, urban or forest sites, which supported our initial expectations that less disturbed landuse areas support greater biodiversity. However, the opposite was true for diversity measures derived from traditional methods, whereby greater biodiversity was observed in agriculture and urban sites, compared to moorlands or acid grasslands. The lower biodiversity observed with traditional methods metrics in the less disturbed sites is likely due, in part, to substrate types. The moorland and acid grassland sites are dominated by large boulders or loose sediment, which are not ideal substrates when performing traditional kicknet sampling methods, that perform better with gravel substrate (significant positive alpha diversity with increased gravel coverage $p=0.007$ ), and may lead to under sampling of the local taxa ${ }^{16}$. Another possible explanation is that the eDNA sampling of these sites may have included a greater surrounding diversity due to eDNA transport dynamics 44,45 . There is, however, no clear indication that the landscape or catchment surrounding these sites is any more diverse, as the moorland sites in particular are at higher elevation and more isolated compared to the other landuse sites. However, the moorland sites do represent more exposed areas compared to forest or agricultural sites, which may allow greater macroinvertebrate diversity to occur, thereby promoting localized diversity and thus increased turnover. The results from eDNA monitoring were more in agreement with common expectation that unmodified landuse should hold greater biological diversity, particularly with regards to the higher diversity found in moorland and acid grassland sites, which were the least modified areas in the catchment. As a recommendation, eDNA sampling is likely to be more beneficial for detecting difficult to identify biomonitoring groups such as Chironomidae or Diptera, as well as across more variable substrate types which could introduce bias when traditional sampling methodologies are used.

The functional diversity of the catchment is largely dominated by collector feeders, indicating that the regional biodiversity assembly is driven by fine particulate organic matter (FPOM), which is also referred to as seston ${ }^{46,47}$. Two main factors contribute to the high FPOM driver in the Conwy. First, the widespread fecal input from animal agriculture that covers the entirety of the catchment. And second, although notably less intensive on the upland moorland sites and the combined effect of the large high elevation moorland ${ }^{48}$ and periodic flash flooding history associated with the catchment, that will frequently deposit large volumes of FPOM into the lower elevation systems. Whereas the moorland effect is likely limited to certain headwater sites in this study, the quality difference in FPOM generated from agriculture vs moorland sources likely plays a role in the diversity differences seen between sites where collectors dominate on the whole ${ }^{46,49}$. Seasonal shifts in functional traits were observed with eDNA, but 
not with traditional methods. This is reflected in the ability of eDNA to detect more functional groups compared to traditional methods, particularly with regards to Diptera, including Chironomidae and Simuliidae (blackflies), which are important collector groups that are strongly affected by changes in temperature ${ }^{48}$. Likewise, eDNA functional assessment indicates a change in grazer functionality with season, closely following expectations of periphyton availability, which is a key driver of grazer activity 50 .

With regards to turnover, genera, EPT and Chironomidae showed similar trends whereby the inter-site dissimilarity was significantly attributed to seasonal replacement of species along the environmental gradient. For the eDNA based methodology, there was a greater turnover observed in urban and forest sites, which was attributed to higher $\mathrm{pH}$ and lower moss and boulder coverage compared to other sites. Conversely, traditionally derived turnover was greater in moorland sites, particularly in sites with increased boulder and moss coverages. The disparity in the observed relationship between methods is likely driven largely by the methods themselves and the underlying richness values for each method. Specifically, the moorland communities appear much richer in taxa when using eDNA based methods compared to traditional methodologies. The resulting eDNA derived dynamics may represent a wider community due to the dynamics of eDNA transport as mentioned above, and may also reflect more accurately the expected stability of isolated moorland habitats ${ }^{51}$, which are less impacted by human activities compared to other sites in the study. However, in general terms, both traditional and eDNA based turnover suggest temporal variation in dissimilarity between landuse types, which could be attributed to adaptation (historical) of localized communities to sites ${ }^{4}$.

Nestedness across sites was not significant with either method, suggesting that inter-site dissimilarity was not due to localized environmental sorting as per our initial expectations. The primary driver of observed inter-community heterogeneity was largely due to the seasonal variability and high disturbance frequency of the Conwy drainage ${ }^{36}$, or due to stronger effects of biotic interactions ${ }^{2}$. Most revealingly, eDNA data more accurately depict Chironomidae life cycle patterns compared to traditional sampling. Specifically, seasonal variation in eDNA derived richness follows the expected larval emergence patterns, which increase over spring and summer, and steadily decline over fall and winter ${ }^{27}$, in contrast to traditional sampling which was unable to detect this seasonal shift, possibly due to the difficulty in traditionally observing Chironomidae. Likewise, EPT emergence was detectable via both eDNA and traditional sampling, as Ephemeroptera generally emerge during the spring whilst Trichoptera emerge at various times throughout the year. Additionally, the homogeneity in functional diversity between the landuse sites for both eDNA and traditional based methods further indicated that environmental filtering was not the primary driver of inter-site diversity. The biodiversity across the system is more likely a product of frequent founder and colonizing effects resulting from frequent disturbance patterns, and inability of the sites to establish long-term interacting communities ${ }^{52,53}$.

A wider implication arising from this study is the suggestion that eDNA can disclose a much greater resolution of diversity compared to traditional approaches, and can enable multiple levels of analyses 
from a single data set. Traditional methodologies are not expected to be replaced, but the benefit of comparing traditional findings with eDNA based analyses, which can be analyzed using standardized approaches, is immensely valuable and will help avoid unintended biases introduced from cross study traditional protocols. Overall, our findings show that eDNA is a more effective survey method to sample macroinvertebrates and provides clearer indications of the seasonal and environmental effects on multiple levels of diversity compared to traditional methods. Additionally, we provide a key assessment of regional biodiversity dynamics, which are currently underrepresented in the literature. Specifically, we show that the biodiversity inhabiting the Conwy Catchment, while occurring across a heterogeneous landuse landscape, is not reflective of environmental filtering expectations and is more likely to be driven by adaptations to periodic disturbances. Determining such regional drivers allows for effective biological management, whereby flood control, versus altering current landuse practices, is more likely to have a greater impact on biodiversity in disturbance driven sites.

\section{Declarations}

Acknowledgements

Centre for Ecology and Hydrology for field sampling assistance and macroinvertebrate identification. Genomics Center at University of Birmingham for sequencing services and initial bioinformatics. The project was supported by a Natural Environment Research Council (NERC) Highlight Topic grant (parent grant: NE/N006216/1, relevant subgrants: NE/N005724/1 and NE/N005716/1 United Kingdom).

Data Accessibility Statement

Data including amplicon sequence data, OTU matrices and environmental data associated with the manuscript will be deposited on dryad.

Author Contribution

Designed Research; MS, FE, BC; Performed Research: MS, PS, FE; IB; Analyzed Data MS; Wrote Paper MS; Contributed critically to initial draft of the paper; $\mathrm{FE}, \mathrm{BC}$, IB.

\section{References}

1. Benayas, J. M. R., Newton, A. C., Diaz, A. \& Bullock, J. M. Enhancement of Biodiversity and Ecosystem Services by Ecological Restoration: A Meta-Analysis. Science (80- ). 325, 1121-1124 (2009).

2. Kraft, N. J. B. et al. Community assembly, coexistence and the environmental filtering metaphor. Funct. Ecol. 29, 592-599 (2015).

3. Ovaskainen, O. et al. How to make more out of community data? A conceptual framework and its implementation as models and software. Ecol. Lett. 20, 561-576 (2017). 
4. Ricklefs, R. E. Community diversity: relative roles of local and regional processes. Science (80-. ). 235, 167-171 (1987).

5. Vannote, R. L., Minshall, G. W., Cummins, K. W., Sedell, J. R. \& Cushing, C. E. The River Continuum Concept. Can. J. Fish. Aquat. Sci. 37, 130-137 (1980).

6. Seymour, M., Fronhofer, E. A. \& Altermatt, F. Dendritic network structure and dispersal affect temporal dynamics of diversity and species persistence. Oikos 124, 908-916 (2015).

7. Seymour, M., Deiner, K. \& Altermatt, F. Scale and scope matter when explaining varying patterns of community diversity in riverine metacommunities. Basic Appl. Ecol. 17, 134-144 (2016).

8. Eyre, M. D., Pilkington, J. G., McBlane, R. P. \& Rushton, S. P. Macroinvertebrate species and assemblages in the headwater streams of the River Tyne, northern England in relation to land cover and other environmental variables. Hydrobiologia 544, 229-240 (2005).

9. Donohue, I. et al. Navigating the complexity of ecological stability. Ecol. Lett. 19, 1172-1185 (2016).

10. Flynn, D. F. B., Mirotchnick, N., Jain, M., Palmer, M. I. \& Naeem, S. Functional and phylogenetic diversity as predictors of biodiversity-ecosystem-function relationships. Ecology 92, 1573-1581 (2011).

11. Cardinale, B. J. et al. The functional role of producer diversity in ecosystems. Am. J. Bot. 98, 572592 (2011).

12. Young, R. G. \& Collier, K. J. Contrasting responses to catchment modification among a range of functional and structural indicators of river ecosystem health. Freshw. Biol. 54, 2155-2170 (2009).

13. Moog, O. Fauna Aquatica Austriaca. (Wasserwirtschaftskataster, Bundesministerium fu"r Landund Forstwirtschaft, Umwelt und Wasserwirtschaft, 2017).

14. Koleff, P., Gaston, K. J. \& Lennon, J. J. Measuring beta diversity for presence-absence data. J. Anim. Ecol. 72, 367-382 (2003).

15. Baselga, A. \& Leprieur, F. Comparing methods to separate components of beta diversity. Methods Ecol. Evol. 6, 1069-1079 (2015).

16. Barbour, M. T., Gerritsen, J., Snyder, B. D. \& Stribling, J. B. Rapid bioassessment protocols for use in streams and wadeable rivers: periphyton, benthic macroinvertebrates and fish. 339, (US Environmental Protection Agency, Office of Water Washington, DC, 1999).

17. Deiner, K. et al. Environmental DNA metabarcoding: Transforming how we survey animal and plant communities. Mol. Ecol. (2017). doi:10.1111/mec.14350

18. Bohmann, K. et al. Environmental DNA for wildlife biology and biodiversity monitoring. Trends Ecol. Evol. 29, 358-367 (2014).

19. Epp, L. S. et al. New environmental metabarcodes for analysing soil DNA: potential for studying past and present ecosystems. Mol. Ecol. 21, 1821-1833 (2012).

20. Jo, T., Arimoto, M., Murakami, H., Masuda, R. \& Minamoto, T. Particle Size Distribution of Environmental DNA from the Nuclei of Marine Fish. Environ. Sci. Technol. 53, 9947-9956 (2019). 
21. Moushomi, R., Wilgar, G., Carvalho, G., Creer, S. \& Seymour, M. Environmental DNA size sorting and degradation experiment indicates the state of Daphnia magna mitochondrial and nuclear eDNA is subcellular. Sci. Rep. 9, 12500 (2019).

22. Sassoubre, L. M., Yamahara, K. M., Gardner, L. D., Block, B. A. \& Boehm, A. B. Quantification of Environmental DNA (eDNA) Shedding and Decay Rates for Three Marine Fish. Environ. Sci. Technol. 50, 10456-10464 (2016).

23. Turner, C. R. et al. Particle size distribution and optimal capture of aqueous macrobial eDNA. Methods Ecol. Evol. 5, 676-684 (2014).

24. Sales, N. G. et al. Fishing for mammals: Landscape-level monitoring of terrestrial and semi-aquatic communities using eDNA from riverine systems. J. Appl. Ecol. 57, 707-716 (2020).

25. Seymour, M. et al. Executing multi-taxa eDNA ecological assessment via traditional metrics and interactive networks. Sci. Total Environ. 729, 138801 (2020).

26. Riascos, L. et al. DNA-based monitoring of the alien invasive North American crayfish Procambarus clarkii in Andean lakes (Ecuador). Limnologica 70, 20-25 (2018).

27. Bista, I. et al. Annual time-series analysis of aqueous eDNA reveals ecologically relevant dynamics of lake ecosystem biodiversity. Nat. Commun. 8, (2017).

28. Hänfling, B. et al. Environmental DNA metabarcoding of lake fish communities reflects long-term data from established survey methods. Mol. Ecol. 25, 3101-3119 (2016).

29. Crookes, S. et al. Monitoring the silver carp invasion in Africa: a case study using environmental DNA (eDNA) in dangerous watersheds. NeoBiota 56, 31-47 (2020).

30. Sigsgaard, E. E. et al. Using vertebrate environmental DNA from seawater in biomonitoring of marine habitats. Conserv. Biol. 34, 697-710 (2020).

31. Stat, M. et al. Ecosystem biomonitoring with eDNA: metabarcoding across the tree of life in a tropical marine environment. Sci. Rep. 7, 12240 (2017).

32. Valdez-Moreno, M. et al. Using eDNA to biomonitor the fish community in a tropical oligotrophic lake. PLoS One 14, e0215505-e0215505 (2019).

33. Milner, A. M., Robertson, A. L., Monaghan, K. A., Veal, A. J. \& Flory, E. A. Colonization and development of an Alaskan stream community over 28 years. Front. Ecol. Environ. 6, 413-419 (2008).

34. Álvarez-Cabria, M., Barquín, J. \& Antonio Juanes, J. Spatial and seasonal variability of macroinvertebrate metrics: Do macroinvertebrate communities track river health? Ecol. Indic. 10, 370-379 (2010).

35. McGoff, E., Solimini, A. G., Pusch, M. T., Jurca, T. \& Sandin, L. Does lake habitat alteration and landuse pressure homogenize European littoral macroinvertebrate communities? J. Appl. Ecol. 50, 10101018 (2013).

36. Bussi, G. et al. Climate and land-use change impact on faecal indicator bacteria in a temperate maritime catchment (the River Conwy, Wales). J. Hydrol. 553, 248-261 (2017). 
37. Agency, E. River Habitat Survey in Britain and Ireland: Field Survey Guidance Manual: 2003 Version. (Forest Research, 2003).

38. Spens, J. et al. Comparison of capture and storage methods for aqueous macrobial eDNA using an optimized extraction protocol: advantage of enclosed filter. Methods Ecol. Evol. 8, 635-645 (2017).

39. Leray, M. et al. A new versatile primer set targeting a short fragment of the mitochondrial COI region for metabarcoding metazoan diversity: application for characterizing coral reef fish gut contents. Front. Zool. 10, 34 (2013).

40. Magoč, T. \& Salzberg, S. L. FLASH: fast length adjustment of short reads to improve genome assemblies. Bioinformatics 27, 2957-2963 (2011).

41. Schmieder, R., Lim, Y. W., Rohwer, F. \& Edwards, R. TagCleaner: Identification and removal of tag sequences from genomic and metagenomic datasets. BMC Bioinformatics 11, 341 (2010).

42. R Core Team. R: A Language and Environment for Statistical Computing. (2019).

43. Borcard, D., Gillet, F. \& Legendre, P. Numerical Ecology with R. (Springer, 2011).

44. Deiner, K., Fronhofer, E. A., Mächler, E., Walser, J.-C. \& Altermatt, F. Environmental DNA reveals that rivers are conveyer belts of biodiversity information. Nat. Commun. 7, 12544 (2016).

45. Seymour, M. et al. Acidity promotes degradation of multi-species environmental DNA in lotic mesocosms. Commun. Biol. 1, (2018).

46. Cummins, K. W. \& Klug, M. J. Feeding Ecology of Stream Invertebrates. Annu. Rev. Ecol. Syst. 10, 147-172 (1979).

47. Wallace, J., Hutchens John, J. \& Grubaugh, J. Chapter 12. Transport and Storage of FPOM. in Methods in Stream Ecology 249-271 (2007). doi:10.1016/B978-012332908-0.50014-0

48. Erman, D. C. \& Chouteau, W. C. Fine Particulate Organic Carbon Output from Fens and Its Effect on Benthic Macroinvertebrates. Oikos 32, 409-415 (1979).

49. Miserendino, M. L. \& Masi, C. I. The effects of land use on environmental features and functional organization of macroinvertebrate communities in Patagonian low order streams. Ecol. Indic. 10, 311-319 (2010).

50. Hart, D. D. \& Robinson, C. T. Resource Limitation in a Stream Community: Phosphorus Enrichment Effects on Periphyton and Grazers. Ecology 71, 1494-1502 (1990).

51. Driessen, M. M., Kirkpatrick, J. B. \& Mcquillan, P. B. Shifts in Composition of Monthly Invertebrate Assemblages in Moorland Differed Between Lowland and Montane Locations but not Fire-Ages. Environ. Entomol. 42, 58-73 (2013).

52. Harvey, E., Gounand, I., Fronhofer, E. A. \& Altermatt, F. Disturbance reverses classic biodiversity predictions in river-like landscapes. Proc. R. Soc. B Biol. Sci. 285, 20182441 (2018).

53. Townsend, C. R., Scarsbrook, M. R. \& Dolédec, S. The intermediate disturbance hypothesis, refugia, and biodiversity in streams. Limnol. Oceanogr. 42, 938-949 (1997).

\section{Tables}


Table 1. Environmental site variables (first column) with the associated mean, standard deviation, min and max across sampling sites.

\begin{tabular}{|llllll|}
\hline & Units & Mean & SD & Min & Max \\
\hline $\mathrm{pH}$ & & 6.87 & 0.67 & 5.17 & 7.55 \\
\hline Conductivity & $\mu \mathrm{S} / \mathrm{cm}$ & 92.21 & 69.98 & 25.50 & 252.50 \\
\hline Depth & $\mathrm{cm}$ & 17.87 & 8.84 & 6.33 & 37.00 \\
\hline Moss & $\%$ & 8.61 & 9.46 & 0.25 & 31.25 \\
\hline Algae & $\%$ & 2.32 & 4.31 & 0.00 & 15.00 \\
\hline Plant & $\%$ & 1.16 & 1.26 & 0.00 & 3.75 \\
\hline Boulder & $\%$ & 51.02 & 15.31 & 18.33 & 73.00 \\
\hline Gravel & $\%$ & 42.69 & 12.12 & 26.25 & 65.75 \\
\hline Sand & $\%$ & 6.06 & 4.28 & 0.75 & 18.33 \\
\hline
\end{tabular}

Table 2. Richness statistics for the different levels of richness used in the study including genera, Chironomidae, EPT and functional richness, tested against the set of explanatory variables including landuse type, method (eDNA/traditional), season and the possible two-way interactions. Models shown are the most parsimonious models with the associated model metrics, including degrees of freedom (DF) sum of squares, mean squares, f-value and $p$-value, given for each set of response and explanatory variables. P-values in bold indicate significant effects. 


\begin{tabular}{|c|c|c|c|c|c|c|}
\hline Response & Explanatory & Df & Sum Sq & Mean Sq & $F$ value & p-value \\
\hline \multirow[t]{5}{*}{ Genera } & Landuse & 4 & 4604.0 & 1151.0 & 6.0 & $<0.001$ \\
\hline & Method & 1 & 39759.0 & 39759.0 & 208.4 & $<0.001$ \\
\hline & Season & 3 & 3597.0 & 1199.0 & 6.3 & 0.001 \\
\hline & Landuse:Method & 4 & 11144.0 & 2786.0 & 14.6 & $<0.001$ \\
\hline & Season:Method & 3 & 2843.0 & 948.0 & 5.0 & 0.003 \\
\hline \multirow[t]{5}{*}{ Chironomidae } & Landuse & 4 & 558.6 & 139.7 & 4.9 & 0.001 \\
\hline & Method & 1 & 9573.9 & 9573.9 & 336.5 & $<0.001$ \\
\hline & Season & 3 & 1755.7 & 585.2 & 20.6 & $<0.001$ \\
\hline & Landuse:Method & 4 & 1002.0 & 250.5 & 8.8 & $<0.001$ \\
\hline & Season:Method & 3 & 1329.9 & 443.3 & 15.6 & $<0.001$ \\
\hline \multirow[t]{9}{*}{ EPT } & Landuse & 4 & 20.1 & 5.0 & 1.4 & 0.245 \\
\hline & Method & 1 & 207.6 & 207.6 & 56.5 & $<0.001$ \\
\hline & Season & 3 & 95.9 & 32.0 & 8.7 & $<0.001$ \\
\hline & Group & 2 & 721.4 & 360.7 & 98.1 & $<0.001$ \\
\hline & Landuse:Method & 4 & 220.7 & 55.2 & 15.0 & $<0.001$ \\
\hline & Season:Method & 3 & 29.9 & 10.0 & 2.7 & 0.045 \\
\hline & Season:Group & 6 & 142.2 & 23.7 & 6.4 & $<0.001$ \\
\hline & Landuse:Group & 8 & 90.9 & 11.4 & 3.1 & 0.002 \\
\hline & Method:Group & 2 & 114.0 & 57.0 & 15.5 & $<0.001$ \\
\hline \multirow[t]{9}{*}{ Function } & Landuse & 4 & 1096.0 & 273.9 & 4.7 & 0.001 \\
\hline & Method & 1 & 20726.0 & 20725.5 & 352.9 & $<0.001$ \\
\hline & Season & 3 & 2396.0 & 798.8 & 13.6 & $<0.001$ \\
\hline & Group & 2 & 32941.0 & 16470.6 & 280.5 & $<0.001$ \\
\hline & Landuse:Method & 4 & 7610.0 & 1902.4 & 32.4 & $<0.001$ \\
\hline & Season:Method & 3 & 2164.0 & 721.4 & 12.3 & $<0.001$ \\
\hline & Season:Group & 6 & 690.0 & 115.0 & 2.0 & 0.072 \\
\hline & Landuse:Group & 8 & 613.0 & 76.6 & 1.3 & 0.241 \\
\hline & Method:Group & 2 & 8675.0 & 4337.4 & 66.3 & $<0.001$ \\
\hline
\end{tabular}


Table 3. Linear regression statistics assessing the linear relationship between nestedness and turnover components of beta diversity for eDNA and traditional derived taxon assignments, against the landuse gradient. Bold indicates statistically significant values.

\begin{tabular}{|llllll|}
\hline Response & Method & Estimate & Std. Error & t-value & p-value \\
\hline Genera Nestedness & eDNA & -0.006 & 0.005 & -1.19 & 0.241 \\
\hline Genera Turnover & Traditional & 0.012 & 0.007 & 1.84 & 0.072 \\
\hline EPT Nestedness & eDNA & -0.017 & 0.005 & -3.07 & $\mathbf{0 . 0 0 3}$ \\
\hline Traditional & 0.024 & 0.004 & 6.24 & $<0.001$ \\
\hline EPT Turnover & eDNA & 0.001 & 0.005 & 0.14 & 0.893 \\
\hline \multirow{2}{*}{ Chironomidae Nestedness } & Traditional & 0.004 & 0.008 & 0.56 & 0.576 \\
& eDNA & -0.006 & 0.005 & -1.08 & 0.287 \\
\hline Traditional & 0.032 & 0.005 & 6.90 & $<0.001$ \\
\hline Chironomidae Turnover & eDNA & -0.013 & 0.007 & -1.96 & 0.056 \\
\hline & Traditional & 0.018 & 0.007 & 2.48 & $\mathbf{0 . 0 1 6}$ \\
\hline
\end{tabular}

\section{Figures}




\section{Legend}

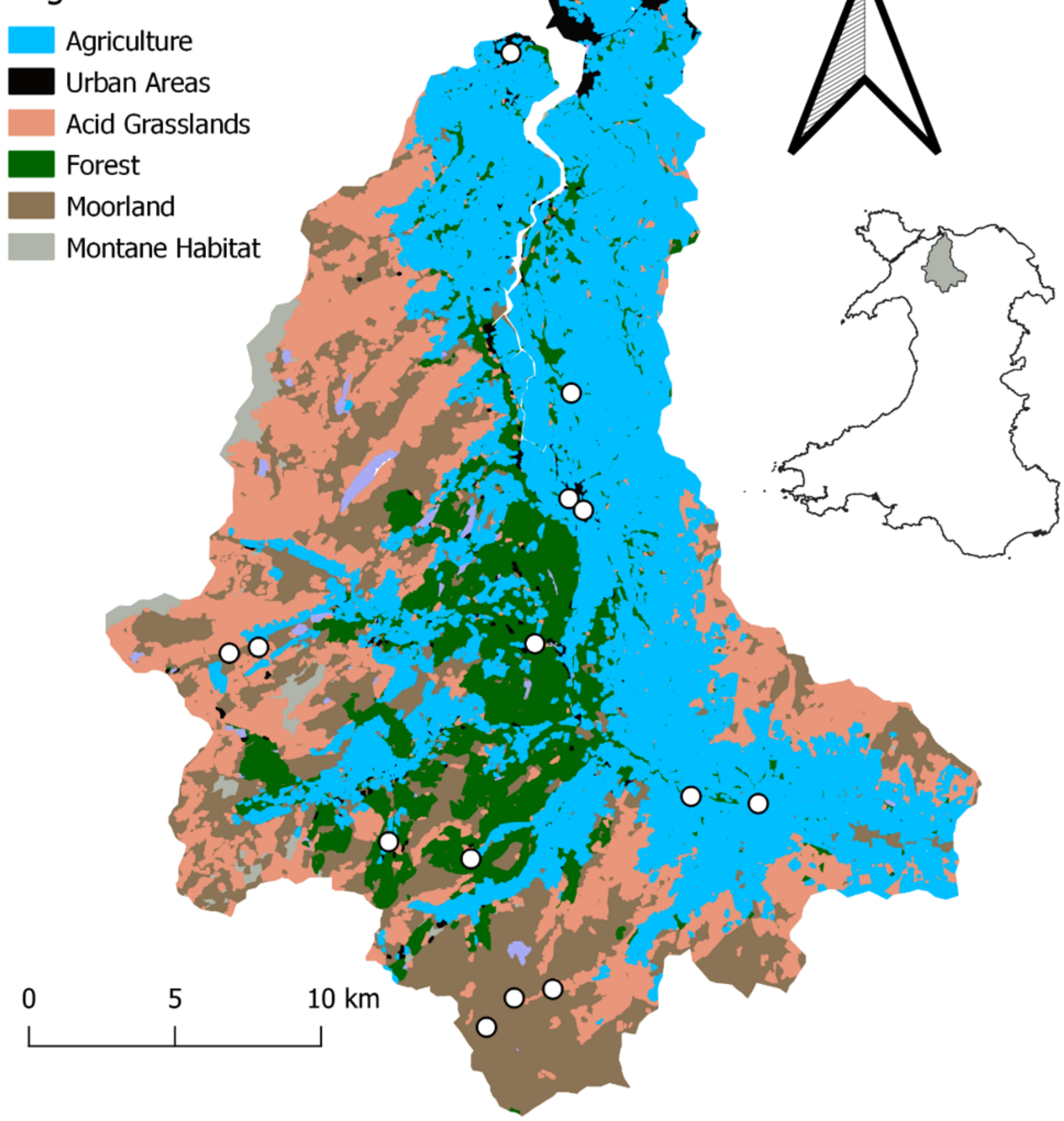

\section{Figure 1}

Map of the study area (Conwy Catchment, North Wales). Colors) colours correspond to different landuse types, including agriculture (blue), bog/moorland (brown), acid grassland (pink), forested (green), and urban areas (black). White circles indicate sampling locations. Note: The designations employed and the presentation of the material on this map do not imply the expression of any opinion whatsoever on the part of Research Square concerning the legal status of any country, territory, city or area or of its 
authorities, or concerning the delimitation of its frontiers or boundaries. This map has been provided by the authors.
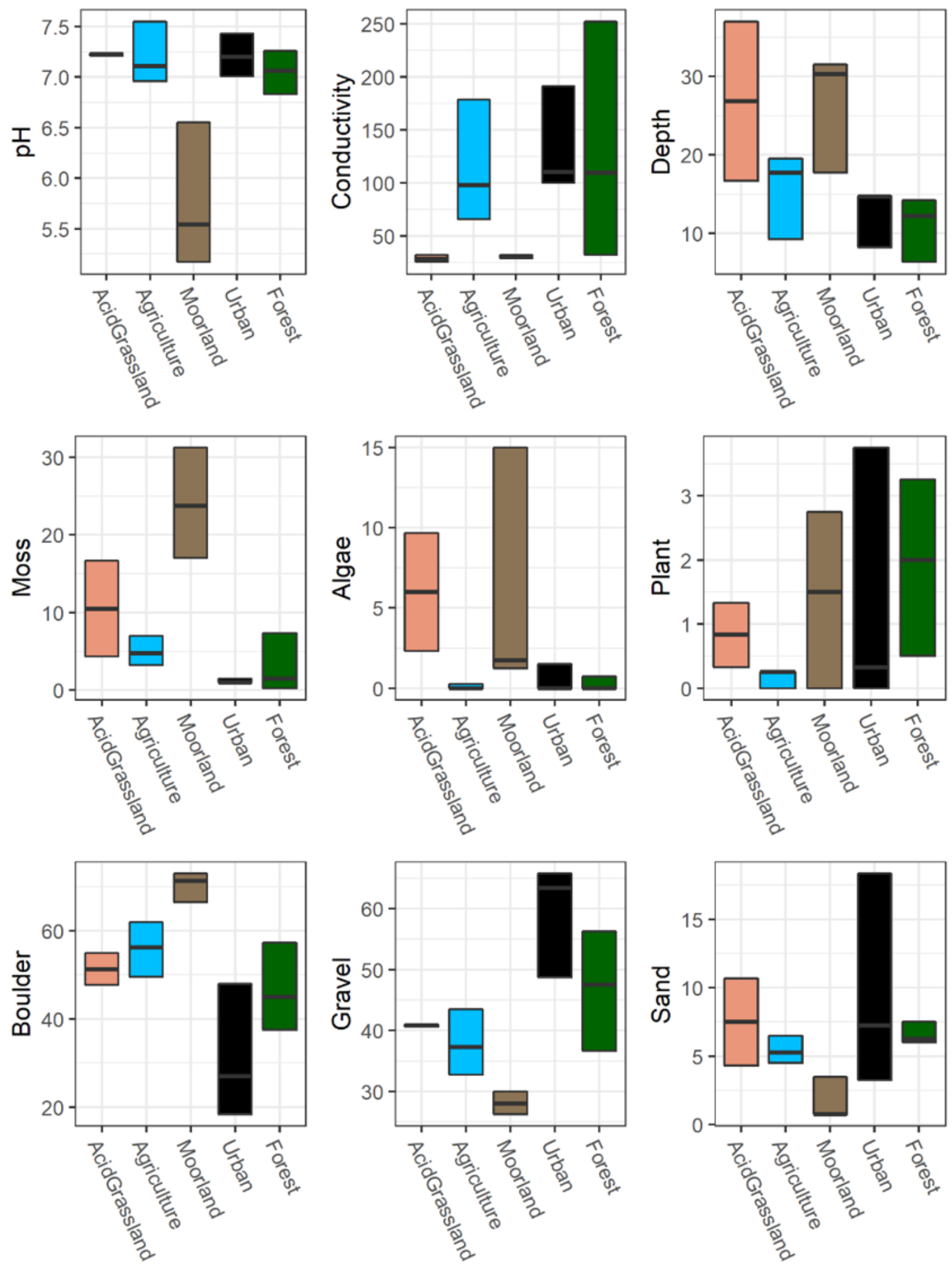

Figure 2

Box plots for each environmental variable (y-axis) sampled in the study, as a single panel. The colors in each plot match the colors in Figure 1 for each landuse type (x-axis). 

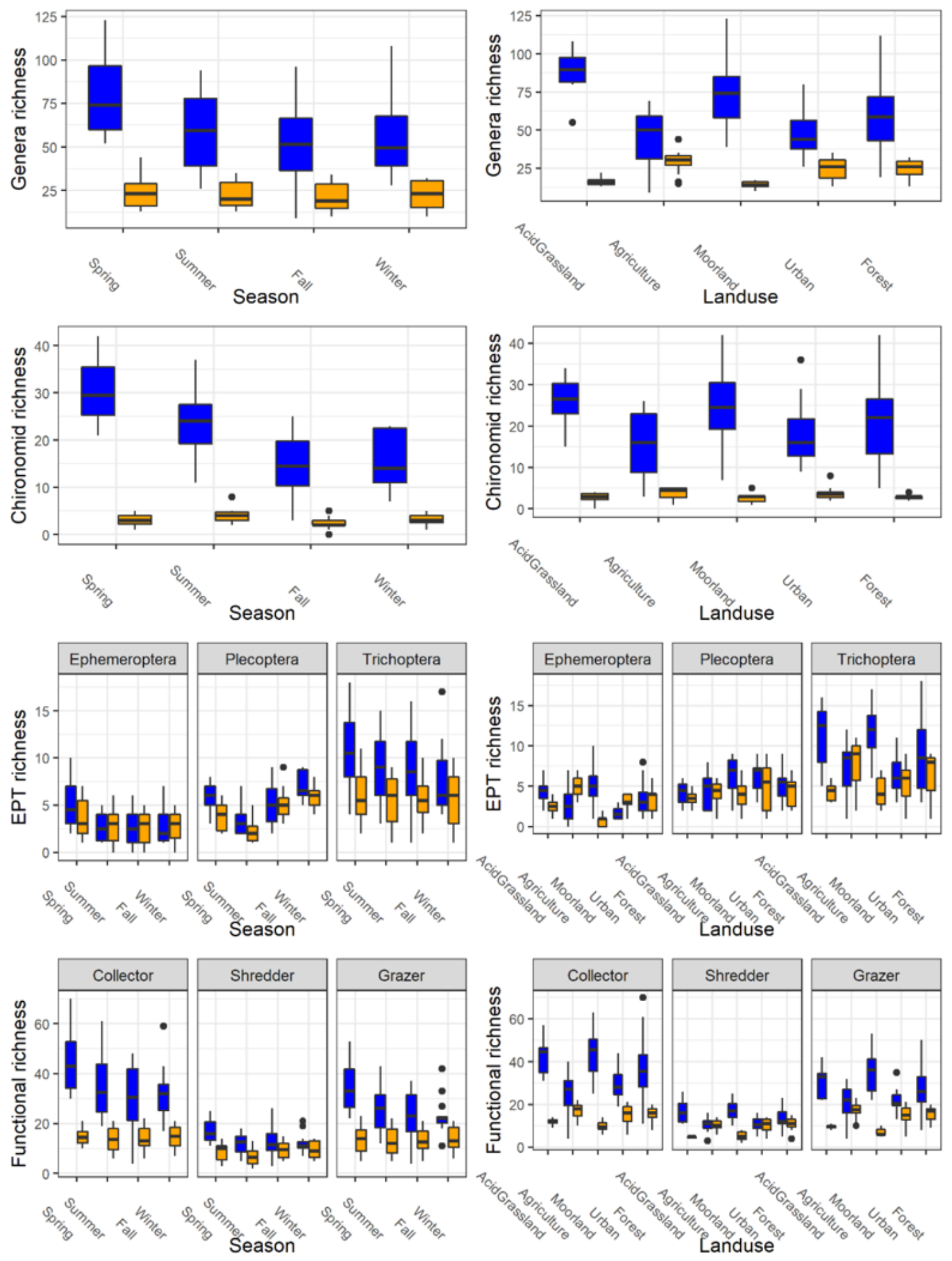

\section{Figure 3}

Box plots showing the richness diversity per season (left panels), and landuse (right panels) for genera (top), Chironomidae (second from the top), EPT (second from the bottom) and functional diversity (bottom). Blue indicates eDNA derived data and orange indicates traditional method derived data. 

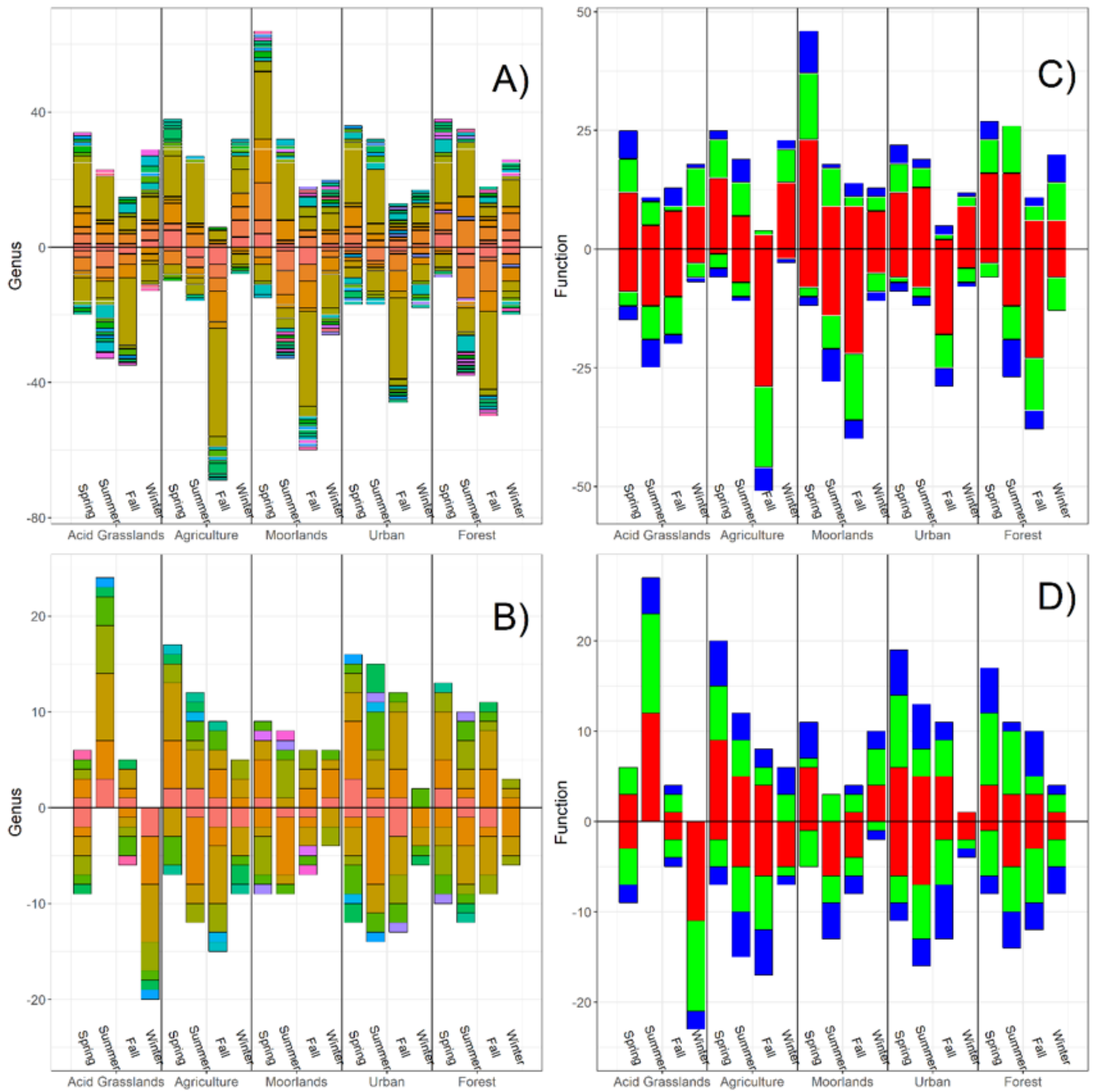

Figure 4

Change in genera richness for eDNA (A) and traditional sampling (B) and the associated change in function for eDNA (C) and traditional (D) sampling. Each subpanel is a unique landuse type showing the sequential change in genera or function whereby values above the line indicate additions in genera or function compared to the previous season and values below the horizontal line indicate loses. Colors for the genera plots are unique classes and each value along the $y$-axis is a unique genus loss or gain. Functional group colors are: scraper $=$ blue, collector $=$ red, gatherer $=$ green . 


\section{Supplementary Files}

This is a list of supplementary files associated with this preprint. Click to download.

- Seymouretalsupplement.docx 